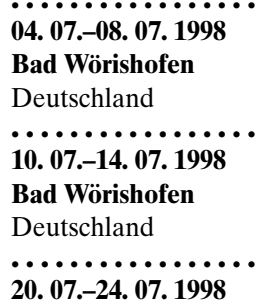

20,07,-24, 07. 1998

München

Deutschland

\section{07.-26. 07.1998}

Neusäss

Deutschland

29.08. 1998

Binningen

Schweiz

.....................

04. 09.-06. 09.1998

Hannover

Deutschland

............

11. 09.-13. 09.1998

Frankfurt

Deutschland

\section{09.-20. 09.1998}

Detmold

Deutschland

................

16. 09.-23. 09.1998

Freudenstadt

Deutschland

\section{09.1998}

Luzern

Schweiz

17..............

17. 09.-20. 09.1998

Hamburg

Deutschland

18....................

18. 09.-20. 09.1998

Essen

Deutschland

\section{09.-27. 09.1998}

Überlingen

Deutschland

Deutschland

\section{Weiterbildungskurs Naturheilverfahren - Kursblock C, Kurs 3}

Weiterbildungskurs Naturheilverfahren - Kursblock C, Kurs 4

Weiterbildungskurs Naturheilverfahren IV

Kurs Ernährungsmedizin - Grundseminar Teil 2

Klassische Homöopathie: Klinisches Seminar - Supervision

Homöopathie in der Augenkunde

Internationaler Homöopathie-Kongress für chronische Krankheiten

\section{Detmolder Herbstwoche für Homöopathie}

Heilfasten im Selbsterlebnis - Lehrinhalte Weiterbildungswoche III Naturheilverfahren

Klassische Homöopathie: Grundkurs Q

Akupunktur-Tage Hamburg: Nadeltechnik - Schröpfen - Moxa

Gesundheitsmesse

3. Wissenschaftlicher Kongress der European Society for Classical Natural Medicine: Projekte «Summer school» (21.-27. 09.) und Bodensee-Konferenz für klassische Naturheilkunde (24.-26. 09.)

\section{ZÄN-Kongress ärztlicher Naturheilverfahren}

Auskunft: KneippÄRZTEbund e. V.,

Postfach 1436, D-86817 Bad Wörishofen Tel. $08247 / 901$ 10, Fax -9 0111

Auskunft: KneippÄRZTËbund e. V., Postfach 1436, D-86817 Bad Wörishofen Tel. 082 47/9 01 10, Fax -9 0111

Auskunft: Akademie Münchener Modell der Erich Rothenfusser Stiftung, Kaiserstr. 9, D-80801 München

Tel. 089/38 88 9833, Fax -393484

Auskunft: Deutsche Akademie für Ernährungsmedizin, Reichsgrafenstr. 11, D-79102 Freiburg Tel. $0761 / 0789$ 80, Fax -7 2024

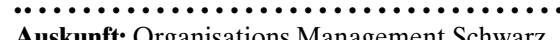
Kehrsitenstr. 23, CH-6362 Stansstad Tel. ++41/41/610 96 86, Fax -2 803036

.....................................

Auskunft: Dr. G. Schwoerer, Tiergartenstr. 126, D-30559 Hannover

Tel. 0511/52 2031, Fax -573608

Auskunft: Verlag Moderne Industrie,

Ecomed Kongress-Service, Frau C. H. Senger,

D-86895 Landsberg

Tel. 081 91/12 55 73, Fax -125600,

E-mail: c.senger@mi-verlag.de

Auskunft: August-Weihe-Institut, Benekestr. 11 . D-32756 Detmold

Tel. $05231 / 34151$, Fax -34152

....................................

Auskunft: Zentralverband der Ärzte für Naturheilverfahren, Alfredstr. 21,

D-72250 Freundenstadt

Tel. $07441 / 21$ 21, Fax -87830

Auskunft: Organisations Management Schwarz, Kehrsitenstr. 23, CH-6362 Stansstad Tel. ++41/41/610 96 86, Fax -2 803036

Auskunft: Deutsche Ärztegesellschaft für Akupunktur, Würmtalstr. 54, D-81375 München Tel. 0 89/7 10 05 11, Fax -7 100525 , E-mail: DAEGfA@t-online.de

Auskunft: Dr. Kater Marketing GmbH, Lindenmannstr. 30, D-40237 Düsseldorf Tel. 02 11/91 45 60, Fax -6724 45, E-mail: info@kater.com

Auskunft: European Society for Classical Natural Medicine (ESCNM), Frau Dr. F. Wilhelmi de Toledo, Organisationskomitee, c/o Klinik Buchinger, PF 1011 65, D-88641 Überlingen Tel. 075 51/8 07/0, Fax -889

Auskunt: Zentralverband der heilverfahren, Alfredstr. 21, D-72250 Freudenstadt Tel. $07441 / 21$ 21, Fax -87830 
…...........

66. 09.-30. 09. 1998

Ettlingen

Deutschland

. 10.1998

Ettlingen

Deutschland

\section{09.-08. 10.1998}

Überlingen

Deutschland

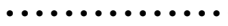

01. 10.-03. 10.1998

Bad Nauheim

Deutschland

-...............

05. 10.-09. 10. 1998

München

Deutschland

\section{10.-15. 10. 1998}

Berlin

Deutschland

02. . . . . . . . .

München

Deutschland

................

05.11 .1998

Luzern

Schweiz

\section{11.-15. 11.1998}

Berlin

Deutschland

\section{11.1998}

Luzern

Schweiz

...............

30. 11.-04. 12.1998

Simbach am Inn

Deutschland

......

04. 12.-06. 12.1998

München

Deutschland

\section{$10.12 .-12.12 .1998$}

Exeter

UK

… . . . . . . .

München

Deutschland

\section{Grundkurs Homöopathie, D/E/F}

Weiterbildungswoche IV mit Heilfasten

Kurs Ernährungsmedizin - Grundseminar Teil 1

Weiterbildungskurs Naturheilverfahren I

\section{Naturheiltage Berlin - Herbstkongress - Kurs I}

\section{Weiterbildungskurs Naturheilverfahren II}

\section{Klassische Homöopathie: Grundkurs S}

\section{Wochenendkongress Naturheilverfahren}

\section{Klassische Homöopathie: Grundkurs T}

\section{Weiterbildungskurs Naturheilverfahren III}

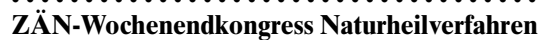

\section{5th Annual Symposium on Complementary Health Care}

\section{Weiterbildungskurs Naturheilverfahren IV}

Auskunft: Akademie für Homöopathie Frau Dr. Ch. Krüger-Winter,

Rosenheimer Str. 113, D-81667 München Tel. 089/6140127

Auskunft: Akademie für Homöopathie, Frau Dr. Ch. Krüger-Winter, Rosenheimer Str. 113, D-81667 München

Tel. $089 / 6140127$

Auskunft: Zentralverband der Ärzte für Natur-

heilverfahren, Alfredstr. 21,

D-72250 Freudenstadt

Tel. $07441 / 2121$, Fax -87830

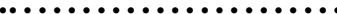

Auskunft: Deutsche Akademie für Ernährungsmedizin, Reichsgrafenstr. 11, D-79102 Freiburg Tel. 07 61/07 89 80, Fax -7 2024

................................

Auskunft: Akademie Münchener Modell der

Erich Rothenfusser Stiftung, Kaiserstr. 9,

D-80801 München

Tel. 0 89/38 889833, Fax -393484

Auskunft: Ärztekammer Berlin, MUT Fortbil-

dung gGmbH, Flottenstr. 28-42, D-13407 Berlin

Tel. 030/40 90 59-42, Fax -40 9059-52

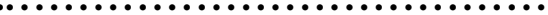

Auskunft: Akademie Münchener Modell der

Erich Rothenfusser Stiftung, Kaiserstr. 9,

D-80801 München

Tel. 089/38 88 9833, Fax -393484

............................

Auskunft: Organisations Management Schwarz,

Kehrsitenstr. 23, CH-6362 Stansstad

Tel. $++41 / 41 / 6109686$, Fax -2803036

Auskunft: Zentralverband der Ärzte für Natur-

heilverfahren, Alfredstr. 21,

D-72250 Freudenstadt

Tel. 07441/2121, Fax -87830

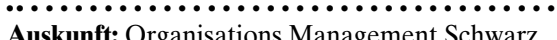

Kehrsitenstr. 23, CH-6362 Stansstad

Tel. $++41 / 41 / 6109686$, Fax -2803036

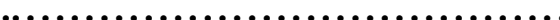

Auskunft: Akademie Münchener Modell der

Erich Rothenfusser Stiftung, Kaiserstr. 9,

D-80801 München

Tel. 089/38 88 9833, Fax -393484

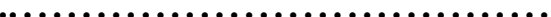

Auskunft: Zentralverband der Ärzte für Natur-

heilverfahren, Alfredstr. 21,

D-72250 Freudenstadt

Tel. $07441 / 2121$, Fax -87830

Auskunft: Symposium Secretariat, Department of Complementary Medicine, Division of Community Health Science, Postgraduate Medical School, University of Exeter, 25 Victoria Park Road, Exeter, Devon EX2 4NT, UK

Auskunft: Akademie Münchener Modell der Erich Rothenfusser Stiftung, Kaiserstr. 9,

D-80801 München

Tel. 089/38 88 9833, Fax -393484 